\title{
A 22-Year-Old Retained Ureteral Stent: One of the Oldest Removed Using a Multimodal Endourologic Approach
}

\author{
Alex Fuselier, MD, Jennifer M. Lovin, MD, Emily F. Kelly, MD, \\ Zachary M. Connelly, $\mathrm{PhD}{ }^{2}$ and Nazih Khater, $\mathrm{MD}^{1}$
}

\begin{abstract}
Background: Ureteral stent encrustation poses a distinct challenge to urologists. The purpose of our study is to present a patient with one of the oldest retained ureteral stents reported in the literature, effectively treated at our institution with a multimodal endourologic approach.

Case Presentation: After IRB approval and patient's consent, we present the case of a 47-year-old man who was referred to our institution for gross hematuria and a right retained ureteral stent, incidentally found on imaging. This patient had a history of traumatic stab wound 22 years prior, requiring an exploratory laparotomy and a ureteral stent insertion. Preoperative CT scan revealed a fragmented and heavily encrusted right ureteral stent. The patient was effectively treated with a multimodal endourologic approach, including a cystolitholapaxy, a right retrograde flexible ureteroscopy (URS), and a prone split-leg right percutaneous nephrolithotomy combined with a right retrograde URS. The patient was rendered stone and stent free.

Conclusion: To our knowledge, this 22-year-old retained stent is one of the oldest reported in the literature. As observed in our patient, multimodal endourologic techniques are safe and effective in patients with retained ureteral stents to render then stent and stone free.
\end{abstract}

Keywords: ureteral stents, retained, shockwave lithotripsy, cystolitholapaxy, ureteroscopy, percutaneous nephrolithotomy

\section{Introduction and Background}

$\mathbf{U}$ RETERAL STENTS HAVE BEEN first described by Zimskind in $1967 .{ }^{1}$ They represent perhaps the most common tool used in a urologist's armamentarium. Stent insertion is performed not only in the context of a wide variety of urologic conditions, mainly stone disease, but also in ureteral malignancies, or any other malignant or benign ureteral compression impairing urinary drainage from the kidney. As with any indwelling foreign object, complications can ensue. When left in place for a prolonged period of time, indwelling ureteral stents may become encrusted. They subsequently pose a distinct and unique challenge to urologists.

Retained stents become difficult to remove, and may require a multimodal endourologic approach that can include the combination of cystolitholapaxy with extracorporeal shockwave lithotripsy, with or without ureteroscopy (URS), and could ultimately require a percutaneous nephrolithotomy (PCNL). We frequently encounter patients with retained forgotten stents for a 6- to 12-month period at our institution.
Consistent with the literature, patients less frequently have stents entombed for 1-5 years, and rare cases of stents retained for $>5$ years.

The purpose of this report is to present a patient with a 22-year-old retained ureteral stent, one of the oldest reported in the world. It was effectively removed at our institution using a multimodal endourologic approach.

\section{Case Presentation}

A 47-year-old man was referred to our institution for dysuria and episodic gross hematuria, with a suspected right retained fractured ureteral stent, incidentally found on kidney, ureter, and bladder radiograph (KUB) (Fig. 1a, b) for work-related injury. The patient had reported a history of traumatic stab wound 22 years prior, requiring an exploratory laparotomy and a right ureteral stent insertion performed in 1996. The patient had no genitourinary symptoms until developing gross hematuria and dysuria 1 week before his presentation to us.

\footnotetext{
${ }^{\mathrm{l}}$ Department of Urology, Louisiana State University Health Sciences Center Shreveport, Shreveport, Louisiana, USA.

${ }^{2}$ School of Medicine, Louisiana State University Health Sciences Center Shreveport, Shreveport, Louisiana, USA.
} 

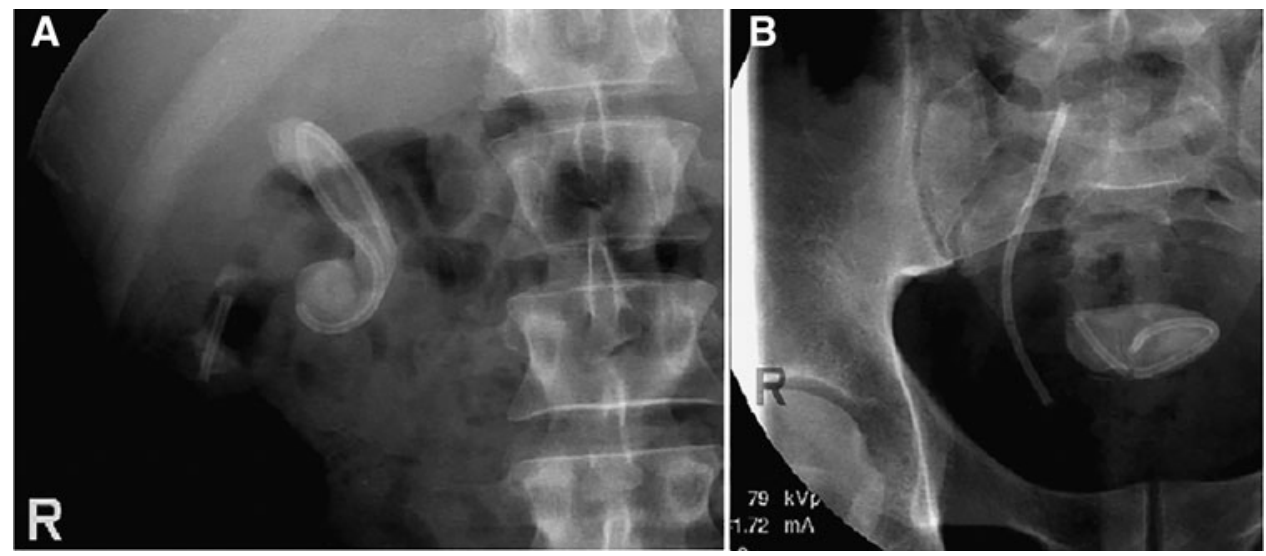

FIG. 1. (a) Proximal stent fragment and encrustation on KUB. (b) Distal stent fragments and large bladder stone on KUB. KUB, kidney, ureter, and bladder radiograph.

The patient has a past medical history of hypertension and chronic lower back pain. He did not have prior urinary tract infections. He has no other past surgical history except his previous exploratory laparotomy and right ureteral stent insertion in 1996. The patient had no prior attempt at stent removal, and his ureteral stent was self-fragmented over the years. He was a nonsmoker, and the patient has no personal or family history of nephrolithiasis or genitourinary malignancy.

His physical examination was normal, including stable vital signs, and no presence of costovertebral tenderness. His initial work-up included a complete blood count that was normal, and a basic metabolic panel demonstrating a normal creatinine level (creatinine $=0.88 \mathrm{mg} / \mathrm{dL}$ upon presentation). His urinalysis showed large leukocytes and blood, and microscopic analysis revealed red blood cells and few bacteria. The urine culture was negative, and a KUB at that time showed right ureteral stent fragments with heavy proximal and distal encrustation (Fig. 1a, b). His CT scan confirmed the encrusted stent fragments and showed a $1.8 \times 3.5 \mathrm{~cm}$ bladder stone (Fig. 2). His CT scan revealed an appropriate and maintained corticomedullary differentiation with no evidence of xanthogranulomatous pyelonephritis. The decision was, therefore, made to preserve the kidney, in the presence of a viable renal function.

\section{Intervention}

To remove the encrusted stent fragments, the patient was subjected to a cystolithotripsy followed by a prone endoscopically combined intrarenal surgery.

The first procedure was a cystolitholapaxy focusing on removing the bladder stone and the most distal stent fragment that was fractured and embedded within the bladder stone. The large bladder stone was fragmented with a $940 \mathrm{~nm}$ laser fiber and crushed mechanically until it has been removed completely off the stent. The laser fiber was inserted through the cystoscope. A cystolithotripsy was performed until the majority of the stone was removed off the stent. Most of the smaller fragments and sediment were irrigated out. However, some harder and larger fragments were remaining despite the initial cystolithotripsy. Our mechanical stone crusher was placed through the sheath of the cystoscope, and under observation, the larger stone fragments were able to be grasped and crushed into smaller passable pieces that were irrigated out through the cystoscope sheath. The rigid cystoscope forceps was used to grasp the end of the distal tip of the fractured stent that was removed from the urethra in its entirety. The cystoscope was again reassembled, and replaced into the bladder, the urethra was noted to have minimal superficial mucosal erythema, and the bladder was free of injury. The bladder was irrigated using the Ellik evacuator and the smallest stone fragments and sediment were irrigated out. Upon inspection of the trigone, some

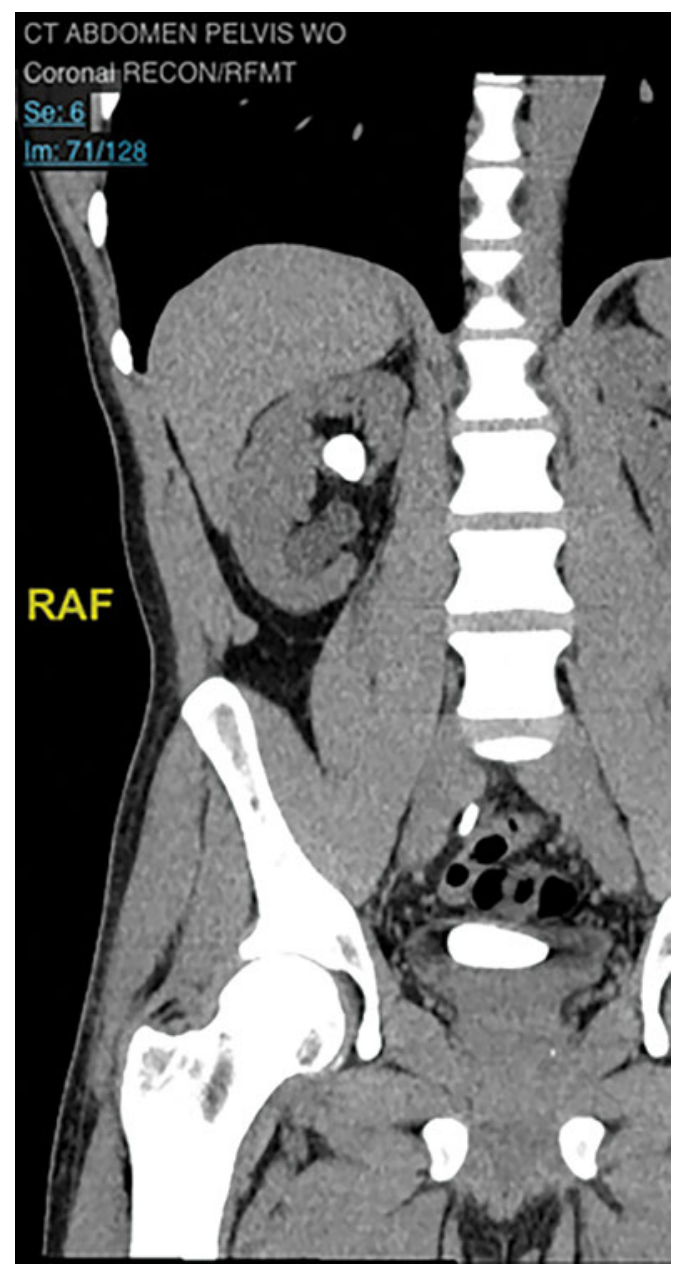

FIG. 2. CT scan showing $\mathrm{R}$ kidney upper pole stone (largest stone was $4.3 \times 0.8 \mathrm{~cm}$ ) and $1.8 \times 3.5 \mathrm{~cm}$ bladder stone. 


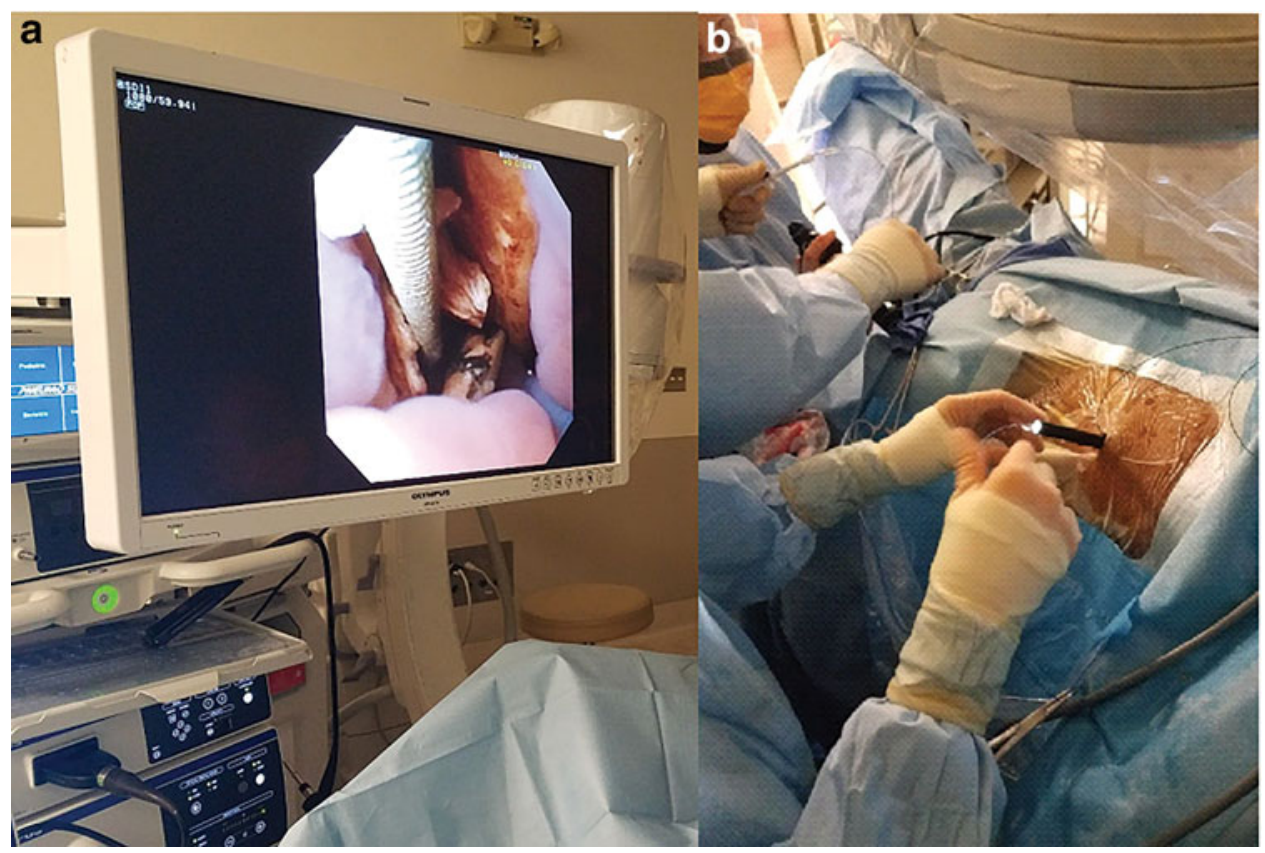

FIG. 3. (a) Right PCNL with rigid nephroscopy revealing proximal part of the fragmented ureteral stent. Heavy encrustations are seen, in the presence of surrounding kidney stone in the right renal pelvis as well. A single floppy super-stiff wire has been previously inserted in an antegrade manner, seen partially in the right lower corner of the picture. Another wire (safety wire) has been also inserted in an antegrade manner (green standard wire seen partially in left upper corner). (b) Antegrade and retrograde access are both performed during the right PCNL. The picture shows flexible ureteroscopy performed from below with basketing of the last remaining small stone fragment upon running the ureter. The tip of the basket with a stone grabbed is seen exiting the sheath at the flank. Full pyeloscopy performed prior revealing no gross residual stone or stent fragment. PCNL, percutaneous nephrolithotomy.

minor "looking-like" squamous metaplasia was noted, as well as surrounding the right ureteral orifice. A scout imaging was taken, and the proximal stent fragment was observed, as well at the distal ureteral fragment. A retrograde pyelogram was performed and showed no significant hydronephrosis, but revealed a torturous ureter with a midureteral narrowing and the distal remnant stent fragment remaining. The distal tip of the stent was briefly observed exiting the ureteral orifice and withdrawn during the retrograde pyelogram. Subsequently, a decision was made to place a $7 \mathrm{~F} \times$ $28 \mathrm{~cm}$ tandem Double-J ureteral stent (Cook Medical Bloomington, IN), over a guidewire under direct vision and fluoroscopic guidance, with the added intent of facilitating a passive dilation of the narrowed ureter. That would help during the upcoming URS allowing an easier retrograde insertion of the ureteroscope.

The second intervention was a right retrograde flexible URS that targeted the fragment emanating from the distal right ureter. A 2.2F N-Circle basket (Cook Medical) was used to extract the distal fragment. The tandem stent was then removed in its entirety. Remaining stone burden and stent fragments were localized in the proximal ureter and renal pelvis.

A right retrograde URS was then attempted using laser lithotripsy to free the stent from encrustation. A small fragment was removed from the central region of the kidney. A larger stent fragment remained heavily encrusted in the renal pelvis, and an antegrade percutaneous renal access was needed to remove the remaining proximal fragment.

A percutaneous access was obtained and a right PCNL was performed using the split-leg table combined with right retrograde flexible URS. The entire residual encrusted proximal stent fragment was removed using rigid nephroscopy (Fig. 3a, b). After removal, flexible nephroscopy along with laser lithotripsy and basket extraction were used to clear the patient's remaining stone burden (Fig. 4).

\section{Discussion}

Ureteral stent placement is a vital tool in the management of a multitude of urologic procedures. However, encrustation

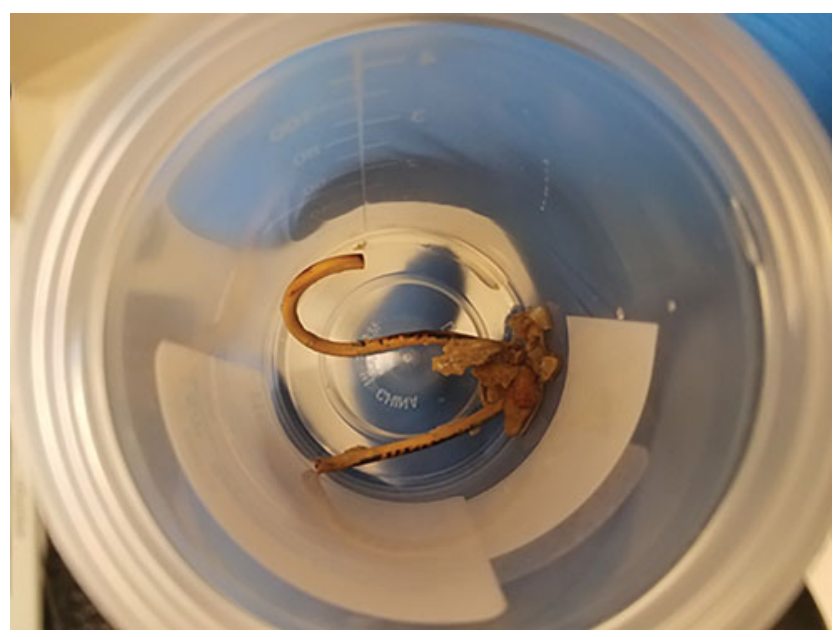

FIG. 4. Proximal part of the fragmented stent completely retrieved through percutaneous access along with stone fragments. 
of ureteral stents can be a cause of morbidity resulting from infection, ureteral obstruction, and stent fragmentation with eventual retention. Our patient had many risk factors for stent encrustation. He was unaware that the stent was placed at the time of initial injury. He tolerated the stent quite well, which made him less likely to follow up with stent-related complaints. He did not live near the medical center that provided initial care, making follow-up difficult.

There are several approaches to the management of encrusted ureteral stents. Similar to our patient, stents with proximal and distal encrustation, including stent fragmentation, usually require multimodal techniques to render the patient stent free. This is consistent with the findings of Weedin et al., ${ }^{2}$ who presented their series of 55 encrusted stents. They confirmed that the amount of encrustation at the proximal curl correlated with the need for multiple procedures. Multimodal endourologic techniques have been shown to be safe and effective in the management of retained stents.

It appears that there is an added difficulty to stent removal when stents are entombed for $>10-20$ years. In our experience, a recently conducted analysis (2010-2018) of patient population who presented to our institution reveals that four patients had retained stents for 4 years, requiring also a multimodality surgical approach. It also appears that having a retained stent $>10$ years may perhaps represent a risk factor for autofragmentation of stent.

The social background of our patient reveals a poor socioeconomic status. He was asymptomatic over the years, living far from our institution, with transportation difficulties, and presented to us after 22 years. In our recent analysis of patients with retained stents, patients traveled a median of 232 Kilometers (19-290) for care. Socioeconomic factors and transportation issues play a major role in extremely long stent retention.

When considering the longest duration of a retained ureteral stent, the literature is scarce. Modh et al. ${ }^{3}$ at the University of Florida reported a 35-year-old stent in the History of Urology Forum at the 2015 annual meeting of the American Urological Association. Our case remains one of the oldest reported in the literature, and was effectively treated with a multimodal endourologic approach.

\section{Conclusion}

To our knowledge, this 22-year-old retained stent is one of the oldest reported in the literature. As observed in our patient, multimodal endourologic techniques are safe and effective interventions for patients with retained ureteral stents to render then stent and stone free.

\section{Disclosure Statement}

No competing financial interests exist.

\section{Funding Information}

No funding was received for this article.

\section{References}

1. Zimskind PD, Fetter TR, Wilkerson JL. Clinical use of long-term indwelling silicone rubber ureteral splints inserted cystoscopically. J Urol 1967;97:840-844.

2. Weedin JW, Coburn M, Link RE. The impact of proximal stone burden on the management of encrusted and retained ureteral stents. J Urol 2011;185:542-547.

3. Modh R, Mason J, Yamamoto A, Stringer T, Bird V. FRII05 The older retained ureteral stent: A history of the ureteral stent. J Urol 2015;193:e589-e590.

$$
\begin{array}{r}
\text { Address correspondence to: } \\
\text { Nazih Khater, MD } \\
\text { Department of Urology } \\
\text { Louisiana State University Health Sciences } \\
\text { Center Shreveport } \\
1501 \text { Kings Highway Room 7-303 } \\
\text { Shreveport, LA 71103-4228 } \\
\text { E-mail: nkhate@1suhsc.edu }
\end{array}
$$

$\begin{aligned} & \text { Abbreviations Used } \\ \mathrm{CT} & =\text { computed tomography } \\ \mathrm{KUB} & =\text { kidney, ureter, bladder radiograph } \\ \mathrm{PCNL} & =\text { percutaneous nephrolithotomy } \\ \mathrm{URS} & =\text { ureteroscopy }\end{aligned}$

Cite this article as: Fuselier A, Lovin JM, Kelly EF, Connelly ZM, Khater N (2020) A 22-year-old retained ureteral stent: One of the oldest removed using a multimodal endourologic approach, Journal of Endourology Case Reports 6:3, 180-183, DOI: 10.1089/ cren.2020.0009. 